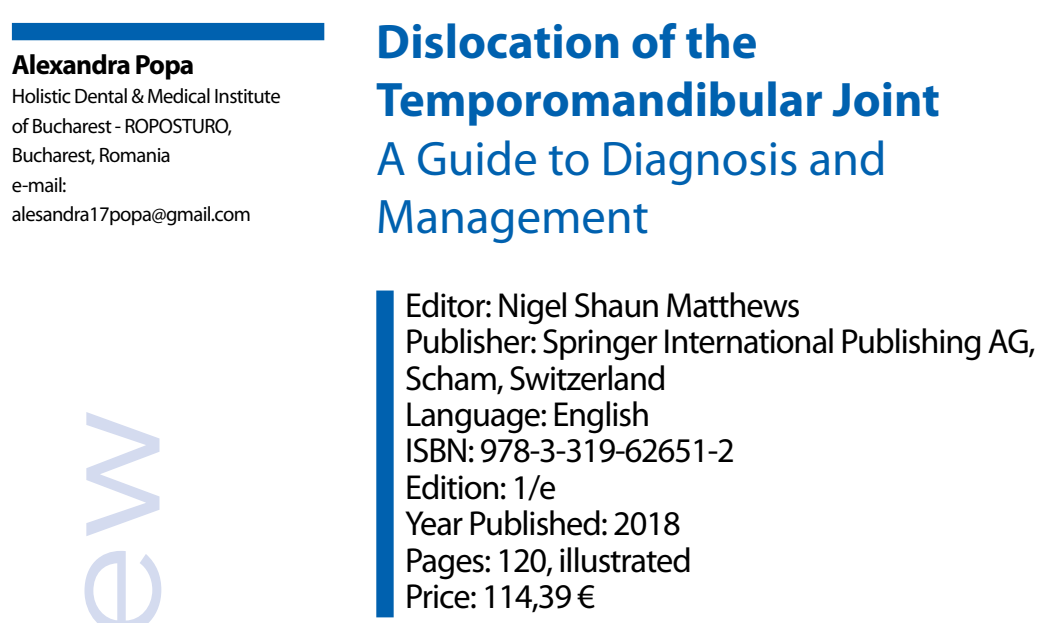

\section{Dislocation of the Temporomandibular Joint A Guide to Diagnosis and Management}

Editor: Nigel Shaun Matthews

Publisher: Springer International Publishing AG,

Scham, Switzerland

Language: English

ISBN: 978-3-319-62651-2

Edition: 1/e

Year Published: 2018

Pages: 120, illustrated

Price: $114,39 €$

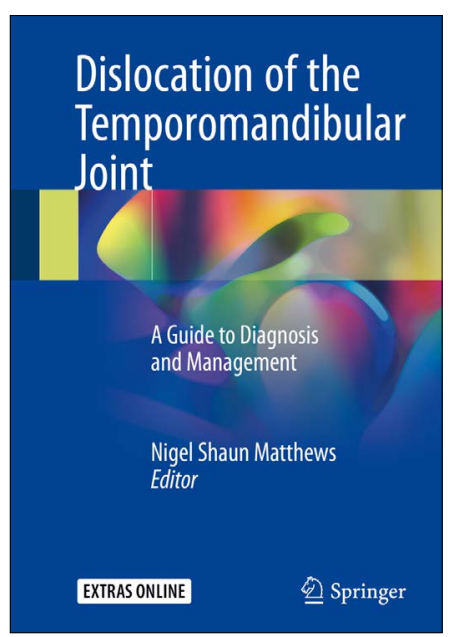

Temporomandibular joint (TMJ) dislocation affects up to $5 \%$ of the population. It is a medical emergency that the dentist must identify as quickly and efficiently as possible to prevent the need for corrective surgery.

The book entitled "Dislocation of the temporomandibular joint. A Guide to Diagnosis and Management" by Professor Nigel Shaun Matthews from the University of North Carolina is a global overview of TMJ dislocation, from issues related to etiology and diagnosis, to management.

The book comprises 9 chapters.

First, we are informed about the etiology, presentation, and diagnosis, while making a clear distinction between dislocation, subluxation, and normal mandibular translation.

The book includes images of the TMJ from conventional radiography, panoramic radiography, scanography, computed tomography (Cone Beam Computed Tomography (CBCT), Multidetector Helical CT (MDCT) and magnetic resonance imaging, with examples in normal anatomy, as well as in pathologies.

Acute TMJ dislocation and technique of manual reduction are clearly defined, classified and presented.

With respect to chronic TMJ dislocation, after the definition, etiology and diagnosis, as well as management and complications are presented.

The TMJ dislocation aspect of pathology is addressed both conservatively and surgically in separate chapters. TMJ dislocation and repositioning of the dislocated mandible is treated arthroscopically, as well as by surgical technique, using alloplastic TMJ prostheses.

The book is an update of the problematic cases of dislocation of the temporomandibular joint, constituting a useful source of information for both the general dentist and the OMF surgeon.

The Books Review is drafted in the reviewer's sole wording and illustrates his opinions. 